\title{
Enterprise Network Marketing Prediction Using the Optimized GA-BP Neural Network
}

\author{
Ruyi Yang $\mathbb{D}$ \\ School of Business, Anyang Normal University, Anyang 455000, Henan, China \\ Correspondence should be addressed to Ruyi Yang; 01045@aynu.edu.cn
}

Received 16 October 2020; Revised 9 December 2020; Accepted 12 December 2020; Published 21 December 2020

Academic Editor: Wei Wang

Copyright (c) 2020 Ruyi Yang. This is an open access article distributed under the Creative Commons Attribution License, which permits unrestricted use, distribution, and reproduction in any medium, provided the original work is properly cited.

\begin{abstract}
As a brand-new marketing method, network marketing has gradually become one of the main ways and means for enterprises to improve profitability and competitiveness with its unique advantages. Using these marketing data to build a model can dig out useful information that the business is concerned about, and the company can then formulate marketing strategies based on this information. Sales forecasting is to speculate on the future based on historical sales. It is a tool for companies to determine production volume and ensure the balance of product supply and sales. It can help companies make correct business decisions to maximize profits. The neural network can approximate the nonlinear function with arbitrary precision, and the time series prediction model based on the neural network can well reflect the nonlinear development trend of information. Based on the analysis of the shortcomings of the traditional BP network, this paper uses a genetic algorithm with good global search capabilities to improve the neural network. The thought and theory of optimizing the initial weight and threshold of the neural network of the GA algorithm are discussed in detail. While expounding the forecasting method, it uses specific examples to analyze the performance and characteristics of the GA-BP network in the enterprise network marketing forecasting. The results show that the GA-BP neural network is higher than the traditional BP neural network in terms of prediction accuracy and adaptability.
\end{abstract}

\section{Introduction}

The current era is an era of informationization and networking. Information network technology has touched every corner of the world, changing the way people receive and distribute information $[1,2]$. The advancement of information network technology has led to the rapid development of the network economy, especially the emergence of the Internet, which has profoundly affected all aspects of human life. At the same time, network technology also provides an important means for companies to improve their comprehensive competitiveness [3, 4]. Companies use new network technologies to change their business philosophy, business organization, and business methods. Network marketing is a new thing that adapts to the development of network technology and social changes in the network age. It is the Internet, computer communications, and digital exchange media. It implements the creativity and goals of the enterprise in new ways, methods, and concepts and completes product pricing new marketing activities in the process of sales, promotion, distribution, and so forth. For enterprises, Internet marketing is an economical and quick-effective sales method in corporate marketing. It has the function of "making small businesses bigger and large enterprises smaller" [5]. And because of the popularity of the Internet today, Internet marketing has become a very important part of corporate marketing strategy. Online marketing performance evaluation is to use a certain number of quantitative and qualitative indicators to compare with the unified evaluation standards. It should follow certain procedures and use scientific methods to make an objective and fair assessment of the company's regular development capabilities and operating results with accurate comprehensive evaluation and interpretation. Marketing performance evaluation is the prerequisite for marketing decision-making $[6,7]$, whether companies can correctly evaluate their own marketing activities and whether they can find that the current era in marketing activities is an information and 
network era. Information network technology has touched every corner of the world and changed the way people receive and distribute information. The advancement of information network technology has led to the rapid development of the network economy, especially the emergence of the Internet, which has profoundly affected all aspects of human life $[8,9]$. At the same time, network technology also provides an important means for companies to improve their comprehensive competitiveness. Companies use new network technologies to change their business philosophy, business organization, and business methods. Network marketing is a new thing that adapts to the development of network technology and social changes in the network age with the emergence of the Internet, computer communications, and digital exchange media $[10,11]$. It implements the creativity and goals of the enterprise in new ways, methods, and concepts and completes product pricing, promotion, distribution, and other processes.

In the process of enterprise network marketing, if the company's future network marketing situation is timely and accurately predicted, it will undoubtedly be more powerful for the company. It can help the company formulate and revise the business plan in time according to the changes in the market and reduce the business risk of the company [12]. At present, the more representative forecasting methods include time series, neural network, and other methods. Since any series can be regarded as an input and output system determined by a nonlinear relationship, the modelling essence of time series forecasting is a nonlinear parameter fitting process. Neural networks can be used for many nonparametric and nonlinear classification and prediction problems $[13,14]$. Adopting the neural network to predict the time series does not need to make assumptions about the characteristics of the sequence in advance, nor does it need to establish precise input and output rules for the system. It is a nonlinear training based on the input set and expected pattern through a self-learning process mapping relationship [15]. According to Kolmogorov's theorem, EBP (error backpropagation) neural network can approximate any rational function with any accuracy. That is, a 3-layer EBP network can complete any $\mathrm{m}$-dimensional to $n$-dimensional mapping [16]. The main purpose of this article is to combine the backpropagation (BP) network and the genetic algorithm (GA) to form a GA-BP network to train and predict time series. This is because of the following:

(1) Each node and weight of a pure BP network will affect the output. Its adaptation process and global approximation process are time-consuming, so the convergence speed of the network is slow. At the same time, the BP network is a nonconvex problem of gradient descent, which is easy to fall into the local minimum value leading to the wrong working mode. Moreover, there is no theoretical guidance on the number of hidden layers and the selection of their units, so BP networks often have great redundancy.

(2) The genetic algorithm is a robust adaptive optimization method based on the principle of biological evolution. This method is based on the sample fitness function and selects, crosses, and mutates the initial population to guide learning and determine the search direction. Because the search is organized by the way of population, it can use random methods to find optimal solutions in multiple areas of the global solution space, so it is particularly suitable for largescale parallel processing [17-19]. Considering the defects of the BP network and the advantages of the GA algorithm, combining the two for training and prediction is a strategy to improve the prediction accuracy. The main technology is initially given the neural network, using the GA algorithm to optimize the initial neural network for weights and thresholds [20]. When the algorithm reaches a certain convergence requirement, the neural network is used for secondary training to avoid local optimization and achieve the purpose of improving network training accuracy and speed. This article uses the GA-BP forecasting method to predict the status of corporate network marketing because the status of corporate network marketing is constantly changing over time. There are many influencing factors, and most of these influencing factors are random. These characteristics are in line with the GA-BP neural network. Therefore, in order to overcome the shortcomings of the traditional predictive model, this paper proposes a genetic algorithm to optimize the BP neural network for predictive analysis of corporate marketing conditions.

The rest of this article is organized as follows. Section 2 analyzes the design of the Internet marketing prediction system. Section 3 establishes an improved BP neural network model of the genetic algorithm with the details. In Section 4, an experiment is carried out and the experimental results are analyzed. Section 5 summarizes the full text.

\section{Design of Internet Marketing Prediction System}

Evaluation indicators are the standards for measuring performance, reflecting the key success factors of corporate network marketing, and revealing the content of corporate network marketing performance evaluation. It is a specific way for the subject of network marketing performance evaluation to fully understand the evaluation object. Therefore, the evaluation index system is the characteristic and connotation of enterprise network marketing performance. A complete and accurate evaluation index system is the basis for scientific and objective evaluation of corporate network marketing performance. The network marketing performance evaluation index system itself must reflect the comprehensive requirements of the enterprise for the management of network marketing activities; it is necessary to conduct a comprehensive and detailed investigation and research on the success factors of network marketing and extract several key success factors from it to form the evaluation index system. When determining the evaluation indicators, we should also follow the principles of combining 
science, purpose, system, practicality, operability, quantity, and quality. As shown in Figure 1, the network marketing performance evaluation indicator system is designed as it has a hierarchical tree structure, which consists of a target layer, a criterion layer, and an index layer.

Website design is mainly a description of the functions, style, and visual design of the website. Website promotion is mainly used to explain how much work network marketers have paid for it, including the number and ranking of registered search engines, the number of links on other websites, the number of registered users, and so forth. The network traffic mainly includes website click-through rate, page views number, and the time each user spends on the site. The financial benefit mainly describes the degree of influence on some financial indicators of the enterprise before and after the use of network marketing. These financial indicators include sales profit growth rate, assetliability ratio, and inventory turnover rate. The publicity benefit mainly describes the impact of the company's use of network marketing on the company's reputation, the company's reputation, and the value of the company's product brand. The competitive benefit mainly describes the company's use of network marketing to improve its competitiveness in the same industry, including the increase in product market expansion rate and product market share. The purchase effect mainly describes the convenience brought to customers by the enterprise network marketing, which reduces the customer's purchase cost. Customer satisfaction describes the degree of customer satisfaction with the enterprise network marketing operation. Customer loyalty describes the company's use of network marketing. The improvement of customer includes loyalty to the company. Advertising costs include online advertising costs, TV advertising costs, newspaper advertising costs, and billboard advertising costs. The marketing management fees include online communication fees, website construction fees, computer time fees, website service fees, and website maintenance fees. Trademark maintenance fee includes a series of costs for maintaining the reputation of a product's trademark. After-sales service fee describes the cost of providing after-sales service to customers.

\section{Improved BP Neural Network Model of Genetic Algorithm}

In the GA search process, there will be problems that will hinder the generation of individuals with high fitness values and affect the GA work. It is a problem that the search direction deviates from the global optimal solution. For such problems, appropriate improved fitness functions and hybrid coding methods are used to solve this problem. This algorithm will also adjust the parameters at the same time, mainly to optimize and improve the crossover operator and mutation operator, crossover probability, and mutation probability.

3.1. Hybrid Coding Scheme. This article combines the advantages of binary encoding and decoding operations with the simplified implementation of genetic operations, such as crossover and mutation, and the advantages of real number coding that are more intuitive, high precision, and no need for decoding when solving continuous parameter optimization problems $[21,22]$. In combination, the purpose of improving the algorithm is achieved. The improved coding method can speed up the genetic operations and perform a large-scale global search, while also solving the continuous parameter optimization problem and improving the optimization accuracy. The algorithm uses the binary coded genes to represent the structure of the BP neural network. That is to say, the binary coded number of hidden layer nodes in the BP neural network and the real coded genes represent the corresponding weights and thresholds, using "structural coding" parallel optimization with "weight coding" and then use the chromosomes of this structure as the operating variables of the genetic algorithm to perform genetic operations. In this way, while optimizing a structure with superior performance, better weight distribution is obtained. The coding scheme is described in further detail below. Assuming that the maximum possible number of hidden layer nodes of a BP neural network is $l$ and the numbers of input and output layer nodes of the network are $m$ and $n$, respectively, the total number of parameters to be optimized in the network is $l \times m+m+m \times n+n+1, l$ represents the number of hidden layer nodes to be optimized. If the number of hidden layer nodes is represented by a string of bits 0 to 1 , the range of the number of hidden layer nodes is 0 to $2^{h-1}$.

The encoding method of all parameters can be visualized as shown in Figure 2. All parameters are divided into five parts, among which the real number coding parts are weights $w_{i j}, v_{i}$ and thresholds $\xi_{i}, \eta_{t}$ and store their respective digits. The design is designed according to the maximum possible number of hidden layer nodes $2^{h-1}$. Reflecting the number of hidden layer nodes indicated in the first part, it will not happen that when there are a relatively large number of nodes in the first part, there are not enough digits in the following four parts to store the weights and thresholds. Since the values shown in the first part are not necessarily the largest possible numbers, this will lead to the possibility of some invalid digits in the following parts. For the convenience of calculation, the effective bit is placed in front of each part, and the invalid bit is placed behind each part. Therefore, when the number of hidden layer nodes is determined, it can be clearly seen that the effective and invalid bits and the crossover and mutation operators need to be valid.

\subsection{Determination of Genetic Operators in Mixed Coding.} This article uses the ranking method as the selection mechanism, which converts the difference in fitness value into order, enlarges (shrinks) the fitness value of an individual, and implies the role of calibration. The selection mechanism is still the probability that an individual with a large fitness value is selected. The larger the value, the smaller the probability. Thus, an individual with a smaller fitness value will be selected. It is suggested to firstly sort the individuals in ascending order according to the fitness value 


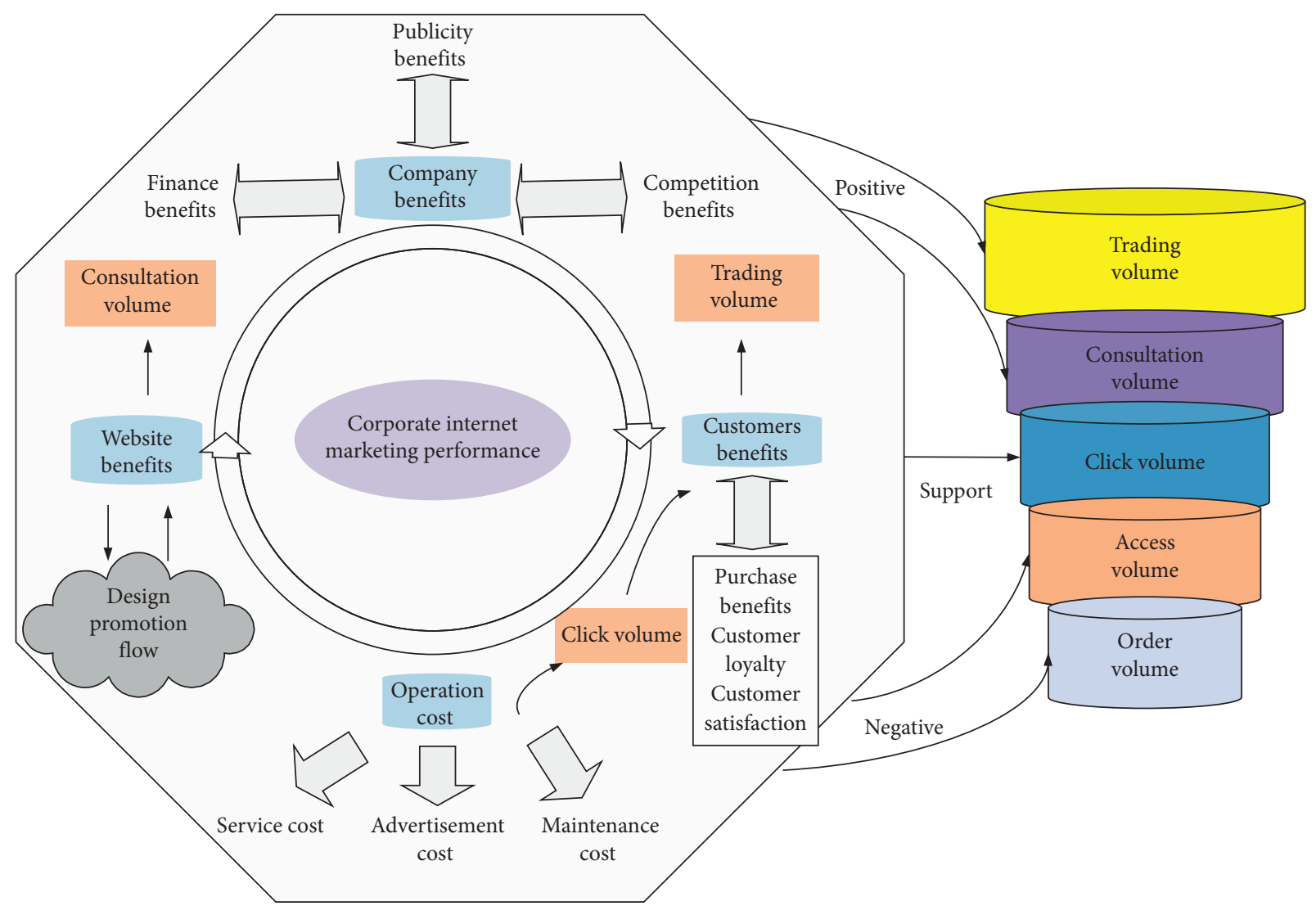

FIGURE 1: Components of corporate marketing.

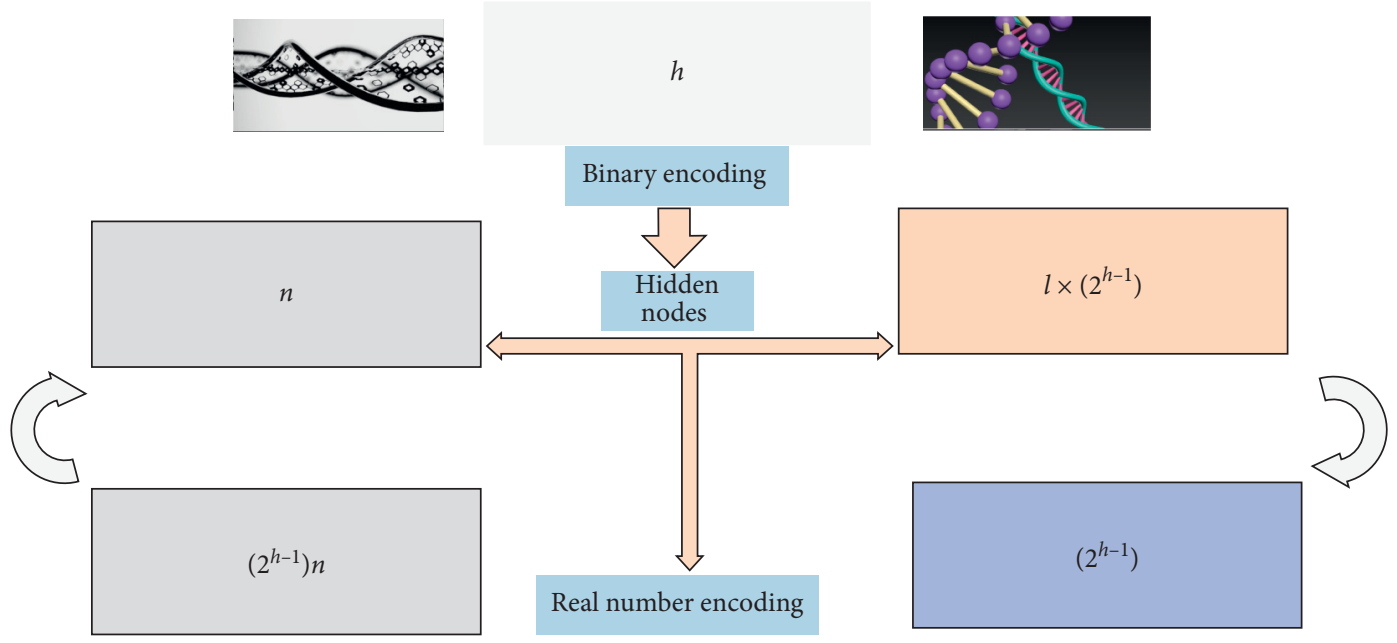

Figure 2: Gene coding and segmentation.

and then calculate the individual's selection probability according to the following formula:

$$
\begin{aligned}
& P_{i}=\frac{\left(1-P_{\max }\right)^{n(i)-1}}{h^{\prime}}, \\
& h^{\prime}=\frac{P_{\max }}{1-P_{\max }},
\end{aligned}
$$

where $p_{\max }$ is the selection probability of the best chromosome and $n(i)$ is the number of the fitness value of chromosome $i$ in the population. The method of proportional selection is used to select each body, calculate the cumulative selection probability $h_{i}(i=1,2, \ldots, L)$ of each body, and generate a random number sequence $r_{j}$ in ascending order in the interval $\left(0, h_{L}\right)$. If $h_{i-1}<r_{j}<h_{i}$, the $i$-th individual to enter the next generation will be selected. 
The crossover operator is the key to ensure that good traits can be inherited. In the binary encoding, this paper uses the single-point crossover method. A crossover point is randomly selected from the two parent strings, and then the corresponding substrings are exchanged. The cross operation of the cross point at the $k$-th position is as follows:

$$
\begin{array}{r}
\left(\alpha_{1} \alpha_{2}, \ldots, \alpha_{m}\right)+\left(\alpha_{1} \alpha_{2}, \ldots, \alpha_{k} \alpha_{k+1}, \ldots, \sigma_{m}\right) \\
\Downarrow \\
\left(\sigma_{1} \sigma_{2}, \ldots, \sigma_{m}\right) \\
\Downarrow \\
\left(\sigma_{1} \sigma_{2}, \ldots, \sigma_{k} \alpha_{k+1}, \ldots, \alpha_{m}\right) .
\end{array}
$$

In the real number coding mode, this article uses arithmetic crossover to calculate. Arithmetic crossover refers to the linear combination of two individuals to produce two new chromosomes. Two individuals $U_{i}^{l}, U_{j}^{l}(l=1,2, \ldots, n)$ in the population are randomly selected to cross. The crossover operator is as follows:

$$
\begin{aligned}
& U_{i}^{l^{\prime}}=\frac{1}{\left(1-c_{i}\right)}\left(U_{i}^{l} c_{i}+U_{i}^{l}\right), \\
& U_{j}^{l^{\prime}}=\frac{1}{\left(1-c_{i}\right)}\left(U_{j}^{l} c_{i}+U_{j}^{l}\right) .
\end{aligned}
$$

In the formula, $U_{i}^{l}, U_{j}^{l}$ are a pair of individuals before the crossover, $U_{i}^{l^{\prime}}, U_{j}^{l^{\prime}}$ are the individuals after the crossover, and $c$ is a uniformly distributed random number in the interval $[0,1]$. Such a cross operation method can obtain many possible results, which can fully realize the information exchange between two individuals and is conducive to finding the global optimal value.

3.3. Improved Genetic Algorithm. Since the genetic algorithm is a probabilistic adaptive iterative optimization process, it has shown good global search performance, which is not easy to fall into local minima. Even if the defined fitness function is discontinuous and irregular, it has a large probability to find the overall optimal solution, and it is suitable for parallel processing. The search does not depend on the characteristics of gradient information and can be used to optimize the BP neural network. Using the genetic algorithm to optimize the initial weight threshold of BP neural network, it can be searched in a larger range instead of random selection of general initial weight, and then the $\mathrm{BP}$ algorithm to fine-tune the network in this solution space to find the optimal solution or approximate optimal solution can be applied. This not only realizes the complementary advantages of the two, but also exerts the extensive nonlinear mapping ability of neural network and the global search ability of genetic algorithm, accelerates the network learning speed, and improves the approximation ability and generalization ability in the whole learning process.

The mathematical description of the improved genetic algorithm to optimize the BP neural network problem is as follows:

$$
T_{1}(v, w, \varsigma, \eta)=\frac{2}{3} \sum_{i=1}^{M} \sum_{i=1}^{m}\left[y_{i}(t)-y_{i}^{\prime}(t)\right]^{2},
$$

where $T_{1}$ is the total error of the network training samples, $y_{i}(t)$ is the ideal output signal, and $y_{i}^{\prime}(t)$ is the actual output of the network:

$$
y_{i}^{\prime}(t)=f\left(v_{i t} \sum_{i=1}^{M} w_{i j} x_{j}(t)-\varsigma_{i}\right)-\eta_{t} \frac{1}{\left(M-M_{1}\right)},
$$

where $w_{i j}$ is the connection weight from the input layer node to the hidden layer node, $v_{i t}$ is the connection weight value from the hidden layer node to the output layer node, $c_{i}$ is the output threshold of the process neuron, $\eta_{t}$ is the output neuron threshold, and $g$ is the hidden, and $f$ is the activation function of output neuron.

To detect the mean square error of the sample, it represents an estimate of the reliability of the network output data. In order to make the designed network have good generalization ability, $T_{2}$ should be made smaller than a given error $\varepsilon$ to meet the reliability of network output:

$$
T_{2}=\frac{2}{M-M_{1}} \sum_{i=1}^{M} \sum_{i=1}^{m}\left[y_{i}(t)-y_{i}^{\prime}(t)\right]^{2} .
$$

The weight correction amount is expressed as

$$
\Delta w_{i j}(n)=-\frac{1}{2} \eta g(n)+\frac{1}{2} \alpha \Delta w_{i j}(n-1),
$$

where $\eta$ is the adaptive learning rate, $g(n)$ is the gradient of the current error function to the weight, $\alpha$ is the momentum factor, and $n$ is the number of iterations.

The design flow chart of BP neural network based on the improved genetic algorithm is shown in Figure 3. The specific steps to improve the BP neural network of the genetic algorithm are as follows:

(1) An initial BP neural network structure is established by setting the relevant parameters and functions of the network, generating the initial population through encoding, and obtaining the optimal weight threshold through genetic operations such as population optimization, selection, crossover, and mutation.

(2) The BP neural network with the obtained optimal weight threshold is optimized, and the best BP neural network structure that meets the requirements after training is obtained.

\section{Simulation Experiment and Analysis}

4.1. Performance Analysis Comparison. In order to compare the similarities and differences between the improved GA$\mathrm{BP}$ algorithm and the general $\mathrm{BP}$ algorithm, the learning situation of the two algorithms was compared and studied. In the simulation experiment of the two types of networks, the number of samples selected is 520 , and the three-layer network structure is adopted. The number of neurons in the input layer is 2 , the number of neurons in the hidden layer is 


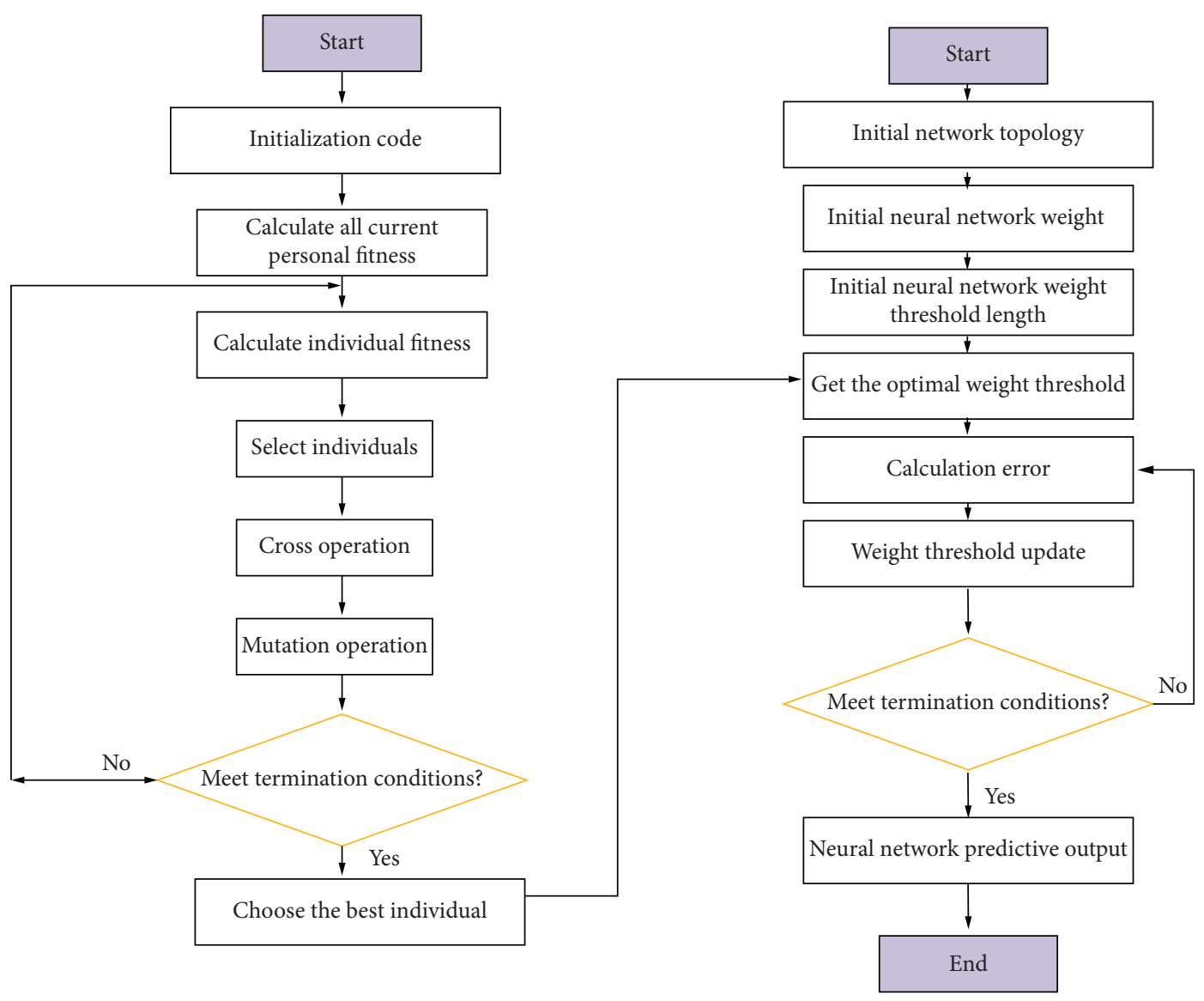

FIgURE 3: The flow chart of the neural network with the improved genetic algorithm.

6 , and the number of neurons in the output layer is 1 . The learning rate $\eta=0.1$ and the difference is that the weights and thresholds of the BP network are initialized by random functions, while the improved GA-BP network algorithm uses the trained optimal individual to initialize the weights and thresholds. Figures 4 and 5 show the curve of the error squared sum fit value obtained by simulation. The solid line in Figure 4 represents the best ideal objective function evolution curve, and the dotted line represents the actual average objective function curve. It can be seen from the figures that after about 300 generations of search, the error of the improved GA-BP algorithm is minimized. The solid line in Figure 5 represents the best ideal fitness evolution curve, and the dotted line represents the actual average fitness evolution curve. After 300 generations of searches, the average fitness of genetic individuals tends to be stable. Figure 6 shows the comparison results of the error sum of squares response curve of the BP neural network without optimization and the BP neural network optimized by the improved genetic algorithm.

4.2. GA-BP Network Forecast Analysis. After repeated training, the number of hidden layer nodes is determined to be 5 . The trained BP neural network model is used to predict the test data set. The results obtained are as follows. It can be intuitively seen from Table 1 and Figure 7 that, based on GA-

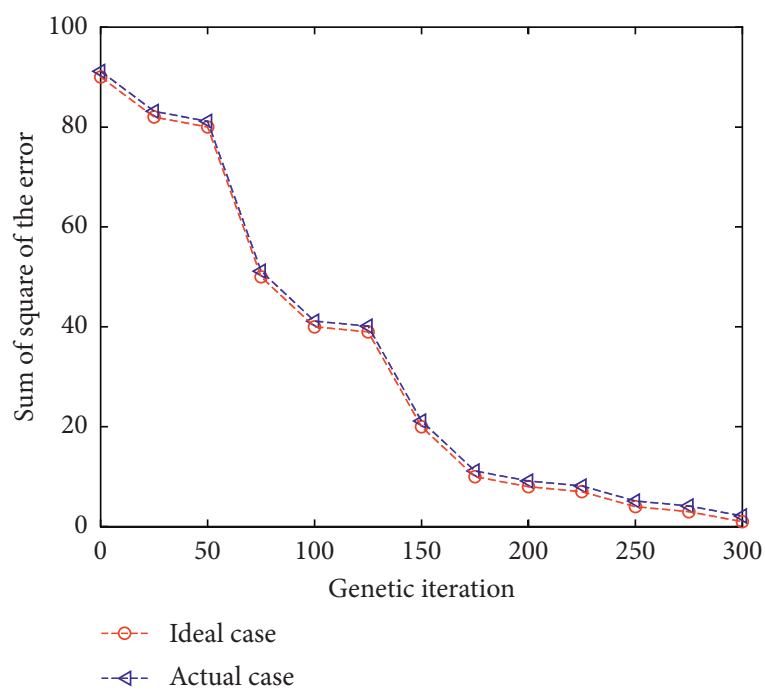

FIgURE 4: The curve of the sum of the square of the error.

$\mathrm{BP}$, the relative error of the neural network sales forecasting model is relatively stable and the error is small, especially when the time series has extreme values.

In order to better analyze the pros and cons of the model, indicators such as mean absolute error (MAE), root mean square error (RMSE), and mean absolute error percentage (MAPE) will be used for model evaluation. These three 


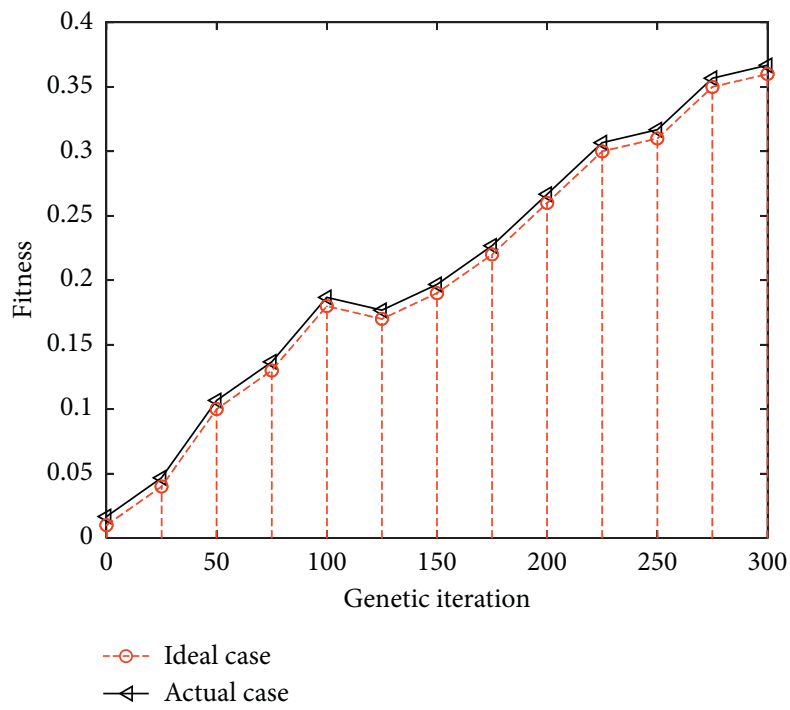

Figure 5: Average fitness curve.

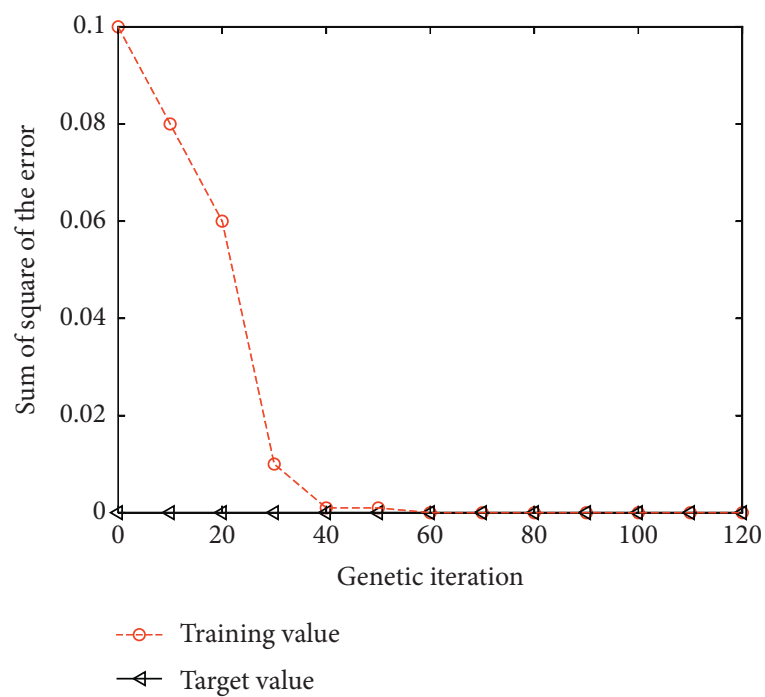

(a)

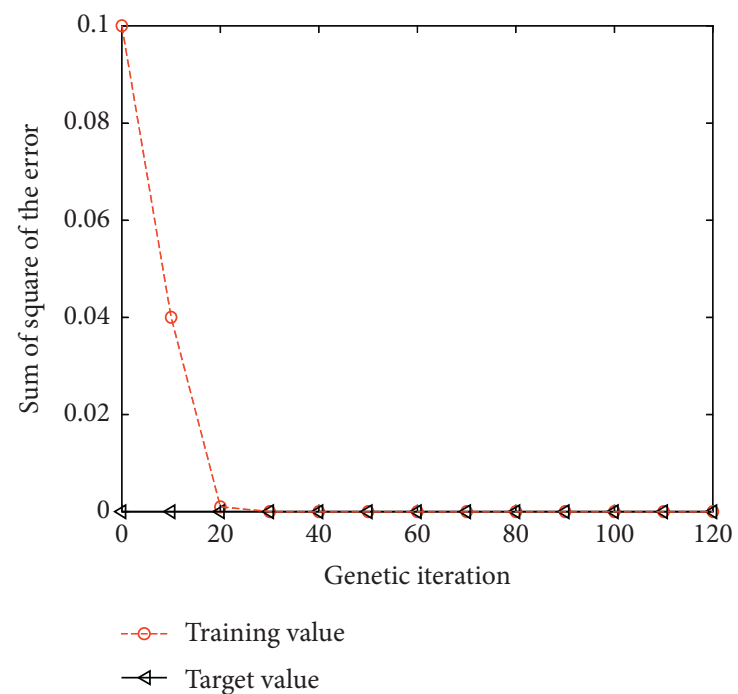

(b)

FIgURE 6: The curves of the sum of the square of the error for the BP neural network: (a) without GA optimization and (b) with GA optimization.

TABLE 1: Comparison of sales forecast results and actual values based on BP neural network.

\begin{tabular}{lccc}
\hline Month & Predicted sales & Actual sales & Relative error (\%) \\
\hline 1 & 302678 & 330955 & 8.54 \\
2 & 159574 & 163534 & 2.42 \\
3 & 200677 & 187598 & -6.97 \\
4 & 220792 & 201755 & -9.44 \\
5 & 218557 & 224954 & 2.84 \\
6 & 209693 & 200996 & -4.32 \\
7 & 235060 & 213466 & -10.12 \\
8 & 207324 & 195249 & -6.18 \\
\hline
\end{tabular}




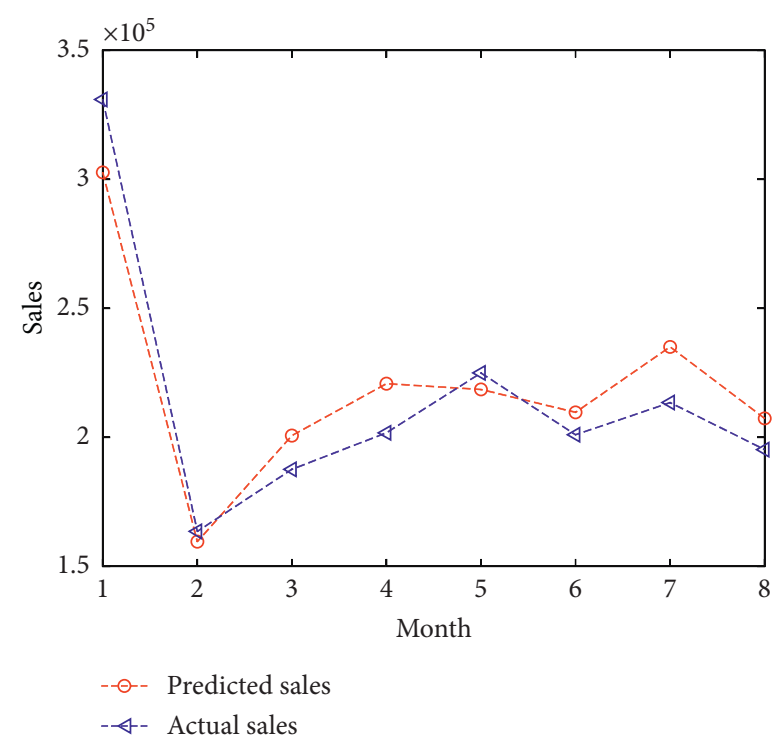

FIgURE 7: Comparison of sales forecast results and actual values based on BP neural network.

TABLE 2: Prediction performances of three types of the prediction model.

\begin{tabular}{lccc}
\hline Prediction model & MAE & RMSE & MAPE (\%) \\
\hline BP & 20101 & 28070 & 8.6 \\
Improved GA & 10600 & 10201 & 4.2 \\
GA-BP & 5600 & 8700 & 2.3 \\
\hline
\end{tabular}

indicators can reflect the size of the error between the predicted value and the actual value. When the value is smaller, the error is smaller. That is to say, the model prediction accuracy is higher. MAE, RMSE, and MAPE are defined as

$$
\begin{gathered}
\text { mean absolute error }=\frac{1}{N} \sum_{i=1}^{N}\left|s_{i}^{\prime}-s_{i}\right|, \\
\text { root mean square error }=\sqrt{\frac{1}{N} \sum_{i=1}^{N}\left(s_{i}^{\prime}-s_{i}\right)^{2},} \\
\text { mean absolute error percentage }=\frac{1}{N} \sum_{i=1}^{N}\left|\frac{s_{i}^{\prime}-s_{i}}{s_{i}}\right| \times M \%,
\end{gathered}
$$

where $s_{i}(t)$ is the ideal output signal and $s_{i}^{\prime}(t)$ is the actual output of the network.

It is the comparison between the predicted value of the model and the true value. Table 2 shows the comparison of the forecasting effects of the forecasting models by MAE, RMSE, and MAPE forecasting evaluation standards. Table 2 and Figure 8 show that, from the three indicators of MAE, RMSE, and MAPE, the evaluation indicators of the GA-BP combined model are the lowest in the model, so the prediction effect is the best. In the original data, there are both linear and nonlinear factors, so a single prediction model, BP neural network prediction model, cannot achieve the desired
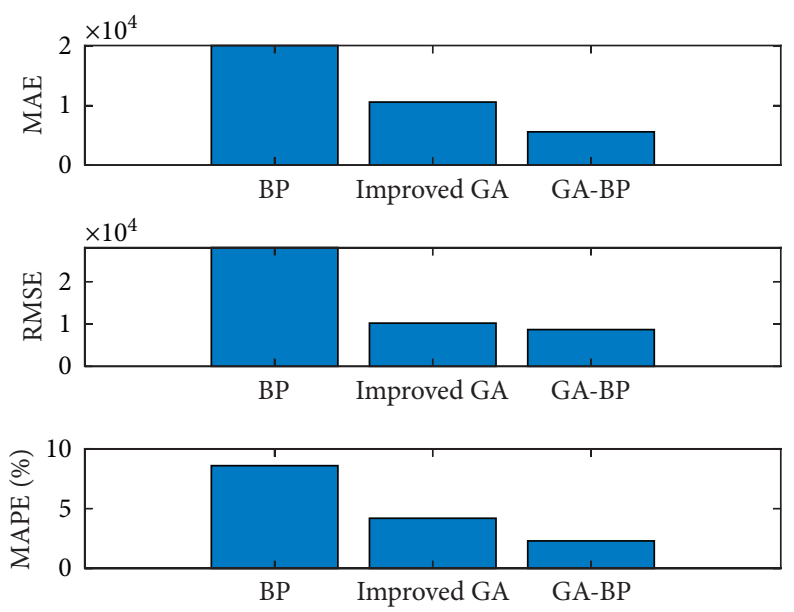

Figure 8: Prediction performances of three types of the prediction model.

prediction effect. The combined model of BP neural network can synthesize the advantages of a single model, can better dig out the complex linear and nonlinear features behind the data, and also improve the prediction accuracy of the model.

\section{Conclusion}

As an emerging industry, network marketing is in the ascendant, and its development speed is amazing. With the country's increasing investment in science and technology 
and the education industry, Internet marketing is gradually becoming the main tool of corporate marketing during the period of high growth in the population of Internet users in my country. Through learning and using network knowledge to flexibly use marketing theory and develop network marketing practice, there is a broad and bright prospect for enterprises to obtain greater benefits. This paper proposes a corporate marketing forecasting model based on a genetic algorithm to optimize the BP neural network, which can effectively overcome the shortcomings of the existing research. In order to further improve the prediction accuracy of the model, an improved genetic algorithm is selected to optimize the BP neural network. Based on the analysis of the shortcomings of the traditional BP network, this paper uses a genetic algorithm with good global search capabilities to improve the neural network. The thought and theory of the GA algorithm for optimizing the initial weight and threshold of the neural network are discussed in detail. While expounding the forecasting method, it uses specific examples to analyze the performance and characteristics of the GA-BP network in the enterprise network marketing forecasting. The results show that the GA-BP neural network is higher than the traditional BP neural network in terms of prediction accuracy and adaptability.

\section{Data Availability}

The data used to support the findings of this study are available from the corresponding author upon request.

\section{Conflicts of Interest}

The author declares no known conflicts of financial interests or personal relationships that could have appeared to influence the work reported in this paper.

\section{References}

[1] F.-M. Yin, "Research on weibo public opinion prediction using improved genetic algorithm based BP neural networks," Journal of Computers, vol. 30, pp. 82-101, 2019.

[2] P. Allemar Jhone, A. M. Sison, and R. P. Medina, "Variable reduction-based prediction through modified genetic algorithm," International Journal of Advanced Computer Science and Applications, vol. 10, pp. 356-363, 2019.

[3] D. Karaboga and E. Kaya, "Adaptive network based fuzzy inference system (ANFIS) training approaches: a comprehensive survey," Artificial Intelligence Review, vol. 52, no. 4, pp. 2263-2293, 2019.

[4] X. Jin, "Research on optimization of intelligent warehousing business of state grid based on genetic algorithm," Journal of Computers, vol. 13, no. 10, pp. 1164-1170, 2018.

[5] O. F. Beyca, B. C. Ervural, E. Tatoglu, P. G. Ozuyar, and S. Zaim, "Using machine learning tools for forecasting natural gas consumption in the province of Istanbul," Energy Economics, vol. 80, no. 1, pp. 937-949, 2019.

[6] Y. Zhang, C. Liu, L. Chen, X. Wang, X. Song, and K. Li, "Energy-related CO2 emission peaking target and pathways for China's city: a case study of Baoding City," Journal of Cleaner Production, vol. 226, no. 1, pp. 471-481, 2019.
[7] P. Ducange, R. Pecori, and P. Mezzina, "A glimpse on big data analytics in the framework of marketing strategies," Soft Computing, vol. 22, no. 1, pp. 325-342, 2018.

[8] F. Ciampi, V. Cillo, and F. Fiano, "Combining Kohonen maps and prior payment behavior for small enterprise default prediction," Small Business Economics, vol. 54, no. 4, pp. 1007-1039, 2020.

[9] M. Bilal, L. O. Oyedele, J. Qadir et al., "Big data in the construction industry: a review of present status, opportunities, and future trends," Advanced Engineering Informatics, vol. 30, no. 3, pp. 500-521, 2016.

[10] T. Song, J. Huang, Y. Tan, and Y. Yu, "Using user- and marketer-generated content for box office revenue prediction: differences between microblogging and third-party platforms," Information Systems Research, vol. 30, no. 1, pp. 191-203, 2019.

[11] Z. Lv, C. Zhong, and D. Jiang, "Guest editorial: smart transportation based on multimedia data mining," Multimedia Tools and Applications, vol. 75, no. 24, pp. 1744317448, 2016.

[12] L. Zhang, Q. Qian, S. Zhang, W. Zhang, and K. Zhu, "A new journal ranking method: the reputation analysis of citation behavior model," IEEE Access, vol. 7, no. 1, pp. 19382-19394, 2019.

[13] Y. Wu, R. Gao, and J. Yang, "Prediction of coal and gas outburst: a method based on the BP neural network optimized by GASA," Process Safety and Environmental Protection, vol. 133, no. 1, pp. 64-72, 2020.

[14] B. Li, X. Tian, and M. Zhang, "Thermal error modeling of machine tool spindle based on the improved algorithm optimized BP neural network," The International Journal of Advanced Manufacturing Technology, vol. 105, no. 1-4, pp. 1497-1505, 2019.

[15] W. Wang, M. Li, R. H. E. Hassanien, M. E. Ji, and Z. Feng, "Optimization of thermal performance of the parabolic trough solar collector systems based on GA-BP neural network model," International Journal of Green Energy, vol. 14, no. 10, pp. 819-830, 2017.

[16] L. Tang, S. Yuan, Y. Tang, and Z. Qiu, "Optimization of impulse water turbine based on GA-BP neural network arithmetic," Journal of Mechanical Science and Technology, vol. 33, no. 1, pp. 241-253, 2019.

[17] Z. Feng and P. Sun, "Medical image segmentation based on GA optimized BP neural network," Investigación Clínica, vol. 60, no. 1, pp. 233-240, 2019.

[18] Y. Liu, X. Zhu, and J. Yang, "Fault diagnosis of PV array based on optimised BP neural network by improved adaptive genetic algorithm," The Journal of Engineering, vol. 2017, no. 13, pp. 1427-1431, 2017.

[19] T. Shen, Y. Nagai, and C. Gao, "Design of building construction safety prediction model based on optimized BP neural network algorithm," Soft Computing, vol. 24, no. 11, pp. 7839-7850, 2020.

[20] Y. Zhu and C. Zhang, "Gold price prediction based on PCAGA-BP neural network," Journal of Computer and Communications, vol. 6, no. 7, pp. 22-33, 2018.

[21] Q. Jiang, R. Huang, Y. Huang et al., "Application of BP neural network based on genetic algorithm optimization in evaluation of power grid investment risk," IEEE Access, vol. 7, pp. 154827-154835, 2019.

[22] Y. Xu and M. He, "Improved artificial neural network based on intelligent optimization algorithm," Neural Network World, vol. 28, no. 4, pp. 345-360, 2018. 